\title{
ARTICLE
}

\section{DNS of MHD Turbulent Flow with Buoyancy}

\author{
Shin-ichi SATAKE ${ }^{1, *}$, Keito FURUMI $^{1}$, Hidenori CHIKAMASA ${ }^{1}$ and Tomoaki KUNUGI ${ }^{2}$ \\ ${ }^{1}$ Dept. of Applied Electronics, Tokyo University of Science, 2641 Yamazaki, Noda, Chiba, 278-8510, Japan \\ ${ }^{2}$ Dept. of Nuclear Eng., Kyoto University, Yoshida, Sakyo, Kyoto, 606-8501, Japan
}

\begin{abstract}
Liquid-metals as coolant material in fusion reactor have a significant role in the design of advanced reactors. The investigation of thermal behavior in the actual facility environment, such as in the case of low Pr number fluid flow, is needed with the buoyancy effect under a magnetic field. In the present study, a direct numerical simulation (DNS) for the low Pr number fluid flow of turbulent heat transfer with buoyancy effect has been carried out under a magnetic field. The values of Hartmann number Ha were 0, 8, 16, 32, 64 and 128, and Prandtl number was 0.06. Grashof number was $4.8 \times 10^{6}$. The turbulent quantities such as the mean temperature, turbulent heat flux, and temperature variant were obtained by DNS. The Reynolds number for channel flow based on friction velocity, viscosity, and channel half width was set to be constant as $\operatorname{Re} \tau=150$. A uniform magnetic field was applied in a direction perpendicular to the wall of the channel. On the large Hartmann number, large-scale thermal convection by the effect of buoyancy can not be found at the whole region of the channel even if Grashof number is large, that is, the thermal transport is restricted by the effect of the magnetic field
\end{abstract}

KEYWORDS: MHD, buoyancy effect, fusion reactor

\section{Introduction}

In recent developments in nuclear fusion research, certain design concepts for liquid breeder blankets for nuclear fusion reactors involve the use of molten salts such as FLiBe as coolant material. ${ }^{1)}$ In addition to FLiBe, liquid-metals are also being studied because they are the conventional coolants and considered to be the best working fluid. Liquid-metals due to their high electrical conductivity have strong magnetohydrodynamic (MHD) that has been found to be very effective in suppressing the turbulence. The mean velocity of liquid coolant in a reactor is strongly influenced by magnetic field, and hence it becomes necessary to understand the behavior of turbulent MHD flow under a magnetic field perpendicular to the direction of flow. Furthermore, because the flow characteristics of coolant with the temperature difference between wall boundary and the coolant are assumed to be different from the usual turbulent MHD flow, it becomes necessary to investigate the buoyancy flow under a magnetic field for the design of an actual working system.

Direct numerical simulations (DNS) of buoyancy-influenced flows in a vertical channel and a horizontal channel were done by Kasagi and Nishimura $(1997)^{2)}$ and Iida et al (2002), ${ }^{3}$ respectively. Their results showed the effect of the orientation of buoyancy force on the behavior of flow and heat transfer. Then, latter investigations revealed that thermal plumes caused by the buoyancy forces were the main contributors to the formation of turbulence. However, the knowledge thus obtained was limited to the understanding of buoyancy only, but buoyancy-influenced flows under a magnetic field were not addressed. Recently, Satake et al

*Corresponding author, E-mail:satake@te.noda.tus.ac.jp
$(2009)^{4)}$ then carried out DNS for heat transfer for low Pr number at the various Grashof numbers under a uniform magnetic field. Their findings concluded that the heat transfer enhancement at turbulent flow with buoyancy effect could be realized even where the flow was low Pr number fluid flow. At present, in fact, very little is understood about the behavior of turbulent heat transfer with the effect of buoyancy under a magnetic field.

In the study being presented here, we applied buoyancy fluid with low Prandtl number to uniform magnetic fields. The objectives of this work were to understand the heat transfer under the combined influence of buoyancy and magnetic field, and to explore the mechanism that can lower the thermal plume via the buoyancy effect under a magnetic field.

Moreover, to address the cooling problem of fusion reactor, where the configuration involves Grashof number under a magnetic field, the design guide also calls for the utilization of low Pr fluid, such as liquid metal. DNSs for the values of Hartmann number have been carried out under a constant Grashof number. We consider the effect of the thermal plume and the magnetic strength.

\section{Computational Conditions of DNS}

In our simulation model a uniform magnetic field $B_{0}$ defines the $y$-axis perpendicular to the streamwise direction that defines the x-axis as shown in Fig. 1. Our DNS code is a hybrid of spectral finite difference method. ${ }^{4)}$ The spectral method is used to compute the spatial discretization in the streamwise and spanwise directions. Nonlinear terms are calculated in physical space and the grid used to compute the nonlinear terms has a resolution 1.5 times finer in these 


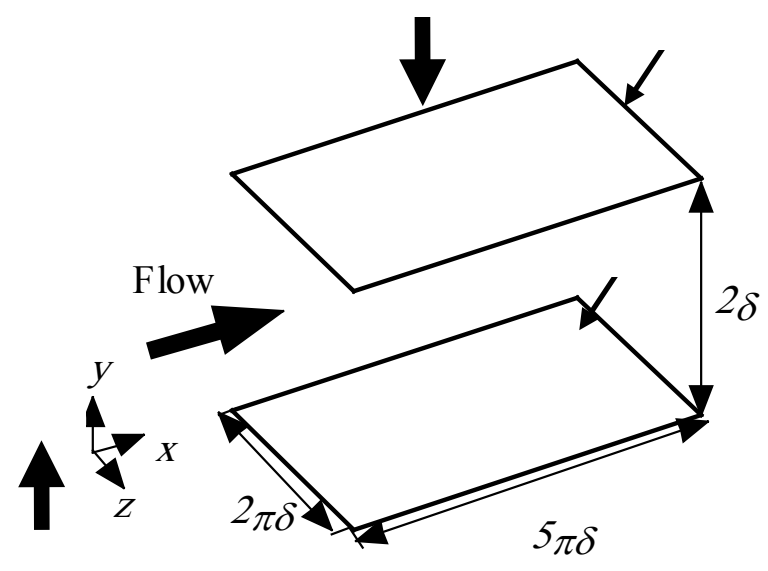

Fig. 1 Computational domain

directions to remove aliasing errors. The derivative in the wall normal direction is computed by a second-order finite difference scheme at staggered grid arrangements. The stream and spanwise components are located at the pressure point. The wall normal component is shifted to half the mesh size. The scalar potential for the electrical field and temperature are also located at the pressure point. The Helmholtz equation for viscous terms and the pressure Poisson equations are solved by using a tri-diagonal matrix technique in Fourier space. The scalar potential and temperature are also solved by the same algorithm. These equations in time are integrated by using the fractional-step method. The second-order Crank-Nicholson scheme applied to the viscous terms is treated implicitly, whereas a modified third-order Runge-Kutta scheme is used for the nonlinear and Lorentz force terms explicitly. Owing to the constant pressure condition, the present simulation was modified for non-dimensional formulation. Here the velocity components were normalized by friction velocity $\sqrt{\tau_{w} / \rho}$ where $\tau_{w}$ is wall shear stress. And for the normalization of the temperature equation, a constant positive temperature difference $\Delta T \equiv\left(T_{b}-T_{t}\right)$ between the bottom and top walls was used. The gravitational acceleration $g$ in the y-direction gives rise to an unstable buoyancy effect. Furthermore, the wall normal component imposes the additional body force according to Bossinessq-approximation. The Grashof number $G r=g \beta \Delta T(2 \delta)^{3} / v^{2}$ was shown in Table 1. All variables and parameter in the governing equations were normalized by the channel half-width $\delta$, the friction velocity $u_{\tau}$ and $\Delta T$. The Reynolds number was kept fixed at $\operatorname{Re}_{\tau}=150$; the number was based the friction velocity $u_{\tau}$ and channel half width $\delta$. The fluid then flowed with a constant pressure. The Neumann condition for the electrical potential was adopted at the wall which meant the existence of an insulation was assumed here. The Hartmann numbers $\mathrm{Ha}=\delta B_{0} \sqrt{\sigma / \rho \nu}$ were set to be $0,8,16,32$, and 64 . These numbers were based on the values of the magnetic field, the kinematic viscosity $v$, the electrical conductivity $\sigma$ and the channel half-width $\delta$. Table 1 summarizes the values of Prandtl number, Reynolds number, and Hartmann number. Periodic boundary condi-
Table 1 Grashof, Hartamnn, Prandtl and Reynolds numbers

\begin{tabular}{ccccc}
\hline & $G r$ & $H a$ & $P r$ & $R e \tau$ \\
\hline Case 1 & $4.8 \times 10^{6}$ & 0 & 0.059 & 150 \\
\hline Case 2 & $4.8 \times 10^{6}$ & 8 & 0.059 & 150 \\
\hline Case 3 & $4.8 \times 10^{6}$ & 16 & 0.059 & 150 \\
\hline Case 4 & $4.8 \times 10^{6}$ & 32 & 0.059 & 150 \\
\hline Case 5 & $4.8 \times 10^{6}$ & 64 & 0.059 & 150 \\
\hline Case 6 & $4.8 \times 10^{6}$ & 128 & 0.059 & 150 \\
\hline
\end{tabular}

tions were applied to the streamwise (x) and spanwise (z) directions. For the wall-normal direction (y), a non-uniform mesh spacing was employed that was specified by a hyperbolic tangent function. A non-slip condition at the wall was applied to the velocity components. The numbers of computational grids used in this study were 128, 128, and 128 in the $x$-, $y$-, and $z$-directions. The Prandtl number was 0.06 as is for Lithium, and this number was selected for the working fluid used for this computation. The other reason for selecting this number was because Lithium or $\mathrm{LiPb}$ has been considered for liquid-metal for fusion reactor. Our code had been validated for its numerical accuracy that measured up to the ones with similar DNS results obtained under the constant pressure condition.

\section{Results and Discussion}

Mean velocity profiles normalized by friction velocity $u_{\tau}$, and mean temperature profiles normalized by friction temperature in Cases 1 through 6 are shown in Figs. 2(a) and (b). Satake et al $^{4)}$ reported that for mean velocity profile with the effect of buoyancy under a magnetic field, there was found no logarithmic profile that could point toward any decrease in the channel center. When the Hartmann number increased, the velocity profile in the Cases 4 and 6 were similar to that by Satake et al. ${ }^{4}$ Moreover, even if Grashof number is $4.8 \times 10^{6}$, the flow becomes completely laminar profile and the profiles at the center become flatten. This meant that the turbulent transport became weak although the turbulence increased by the buoyancy effect. A similar tendency can be seen in the mean temperature profile. The mean temperature profile in Case 1 is close to the turbulence profile owing to the high Grashof number flow. Furthermore, the phenomenon is enhanced by the magnetic field, as seen in Case 6 . Moreover, the mean temperature profiles on magnetic effect in Cases 2, 3, 4, and 5, are represented by becoming close to the straight line. Thus, the thermal transfer decreased.

Figures 3(a) and (b) show the Reynolds shear stress and the scalar flux. The cases of applied magnetic fields have the Reynolds shear stress smaller than they have in other cases because the streamwise balance equation is directly affected by the mean component, magnetic stress (not shown here). The similar tendency can be seen at the scalar flux profile. The scalar flux profile decreased, and the phenomenon is especially marked at the channel center. 
(a)

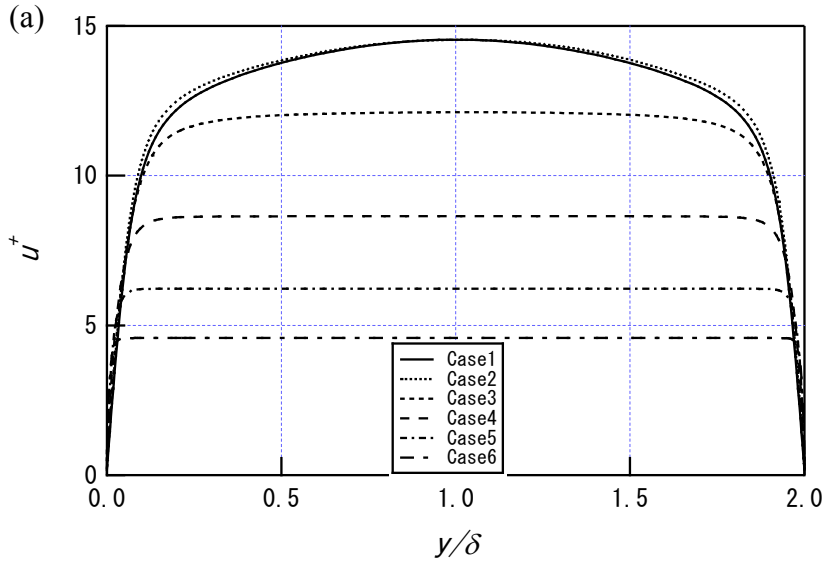

(b)

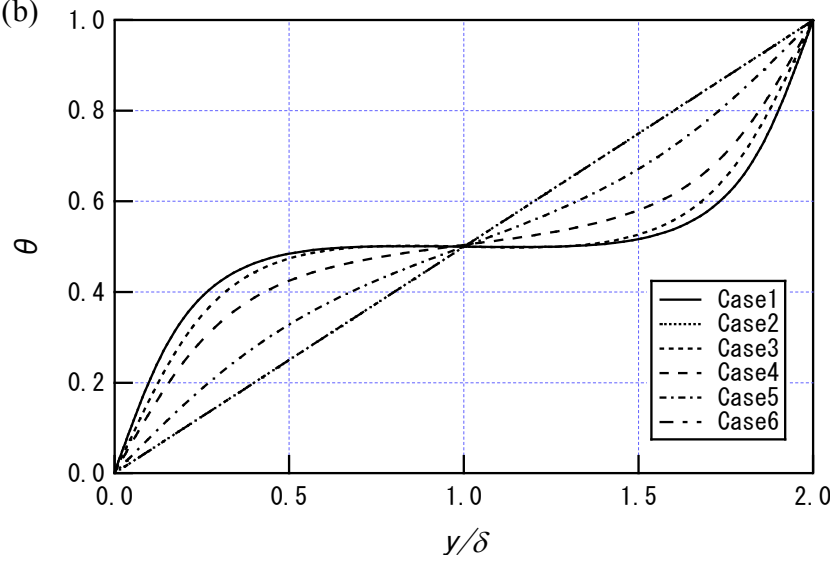

Fig. 2 Mean velocity and temperature profiles
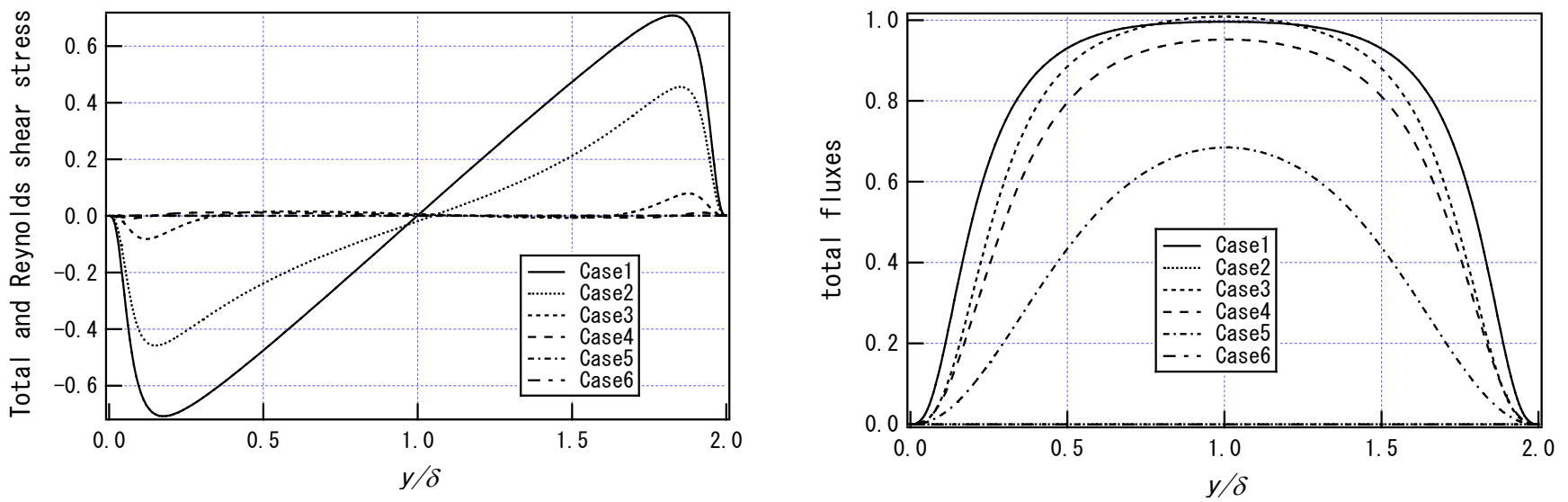

Fig. 3 Reynolds shear stresses and scalar flux

The velocity fluctuations and temperature variance are shown in Figs. 4(a) through (d). Interestingly, the spanwise components for the velocity on the buoyancy increase at the "near wall" region. Especially in Cases 1 and 2 the velocity fluctuation profiles of spanwise component show larger values than those of streamwise component. However, the magnetic strength become larger, the spanwise component decay, the phenomena is enhanced near wall region. Moreover, the wall normal component is attenuated. Thus, it seemed that the existence of the flow motion from the one wall side to the opposite wall side can not be caused by the buoyancy effect. This indicates a phenomenon known as thermal plume and the detail of which will be addressed latter by the aid of visualization. Without the buoyancy effect, temperature variance shows one peak at the channel central region, that is, the temperature variance changed from two peaks to one peaks at the channel center owing to the presence of the magnetic fields.

To examine the phenomenon of varying scales of dominant turbulent structure owing to magnetic field and buoyancy effect, the turbulent structures are visualized in Fig. 5. The nominal visualization lengths for the computational volumes are $5 \pi \delta, 2 \delta$ and $2 \pi \delta$ in the $x$-, $y$-, and $z$-directions. When the lengths are normalized by $v$ and $u_{\tau}$, the full visualized volume is $\left(\mathrm{Lx}^{+}=1570, \mathrm{Ly}^{+}=300, \mathrm{Lz}^{+}=\right.$ 628). Streaky structures in Cases 1 through 3 are shown in
Fig. 5. Without magnetic field in Case 1, many structures and the large scale circulation exist near both walls. Although small fluctuation are not seen in Cases 2, relatively compared with that of Cases 1, the two main large structures still remain and thermal plume exists at the same position. The structures with buoyancy effect are merged, become mainly two scales. The motion is attached from the one side wall to the opposite side wall. This phenomenon is known as thermal plume. Even in the case of applied magnetic field, the high thermal heat transfer still remains owing to the thermal plume. On the other hand in Case 3 in the presence of large magnetic field, the fluctuation decreases and the number of streaks reduce. The thermal plume disappeared complete. Moreover, the structures have a meandering appearance. Note that the streaky structure in Case 4 thought 6 disappeared complete.

\section{Conclusion}

DNS for heat transfer with buoyancy effect was carried out under a uniform magnetic field. Thermal plumes by the effect of buoyancy are found to be in Case 1; streaky structures are found to become larger when the large scale circulation appears. However, at the large Hartman number, the thermal plume decayed and the streaky structure decreased. Therefore, the flow become laminar and the thermal transport also become weak. This means that it is necessary 

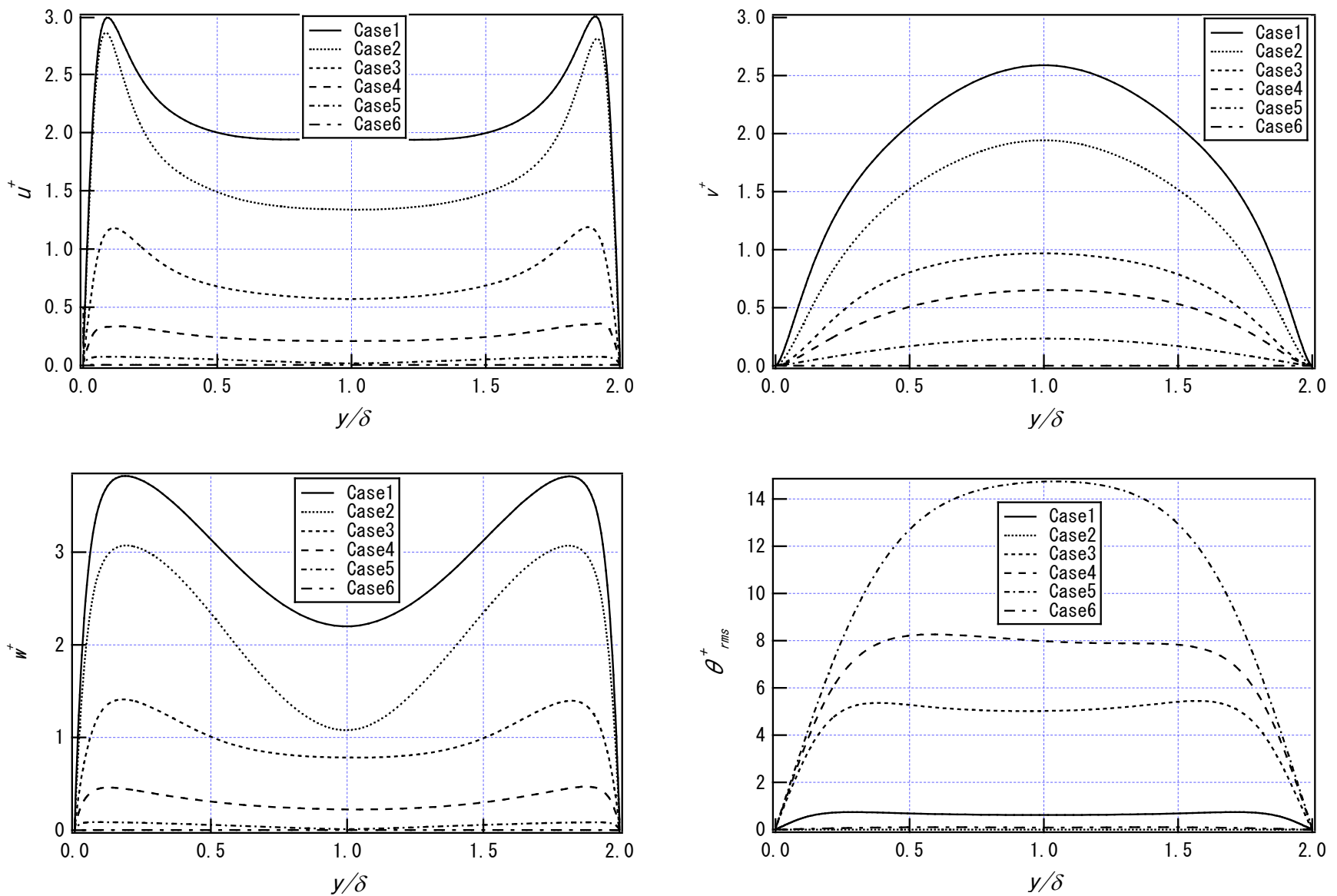

Fig. 4 Velocity fluctuation profiles and temperature fluctuations; (a) Streamwise component, (b) Wall normal component, (c) Spanwise component,

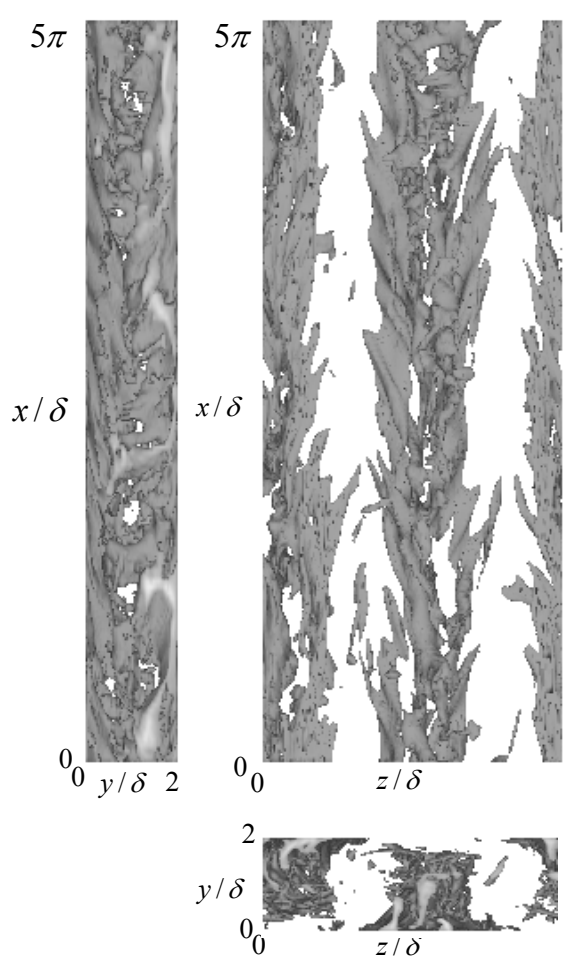

(a)

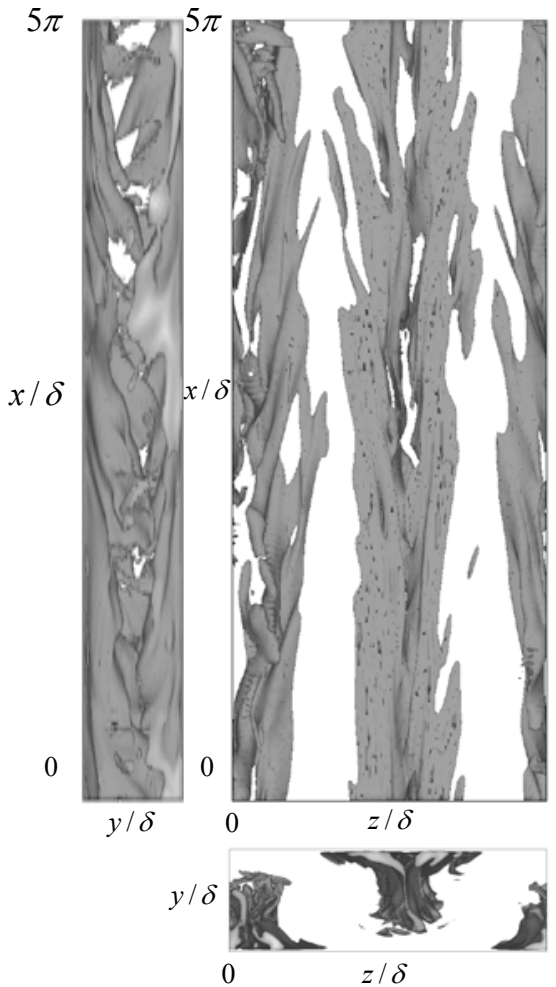

(b)

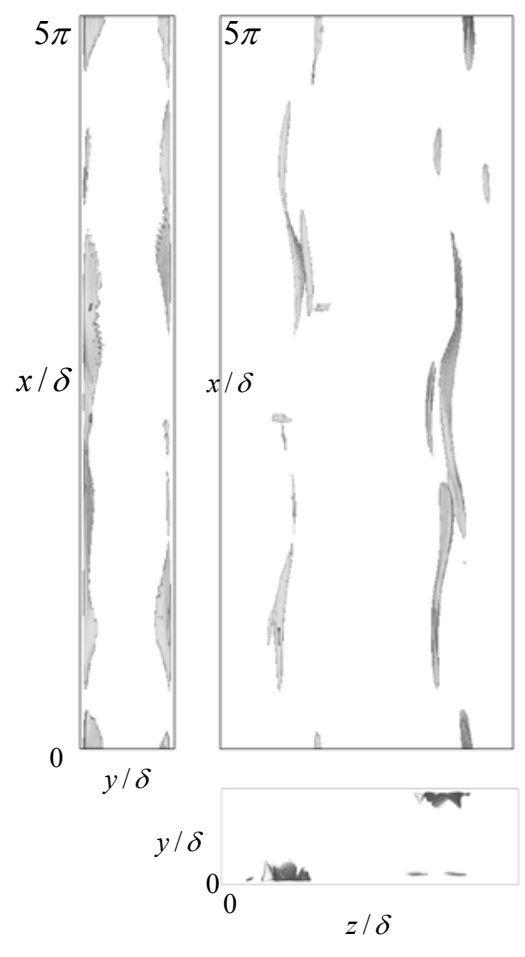

(c)

Fig. 5 Three-dimensional contour surface of low-speed streak, $u^{+}<-3.0:$ (a) Case 1, (b) Case 2, (c) Case 3 
to consider the fusion reactor design of thermal mixing argumentation for buoyancy flow under a magnetic field because the heat transfer enhancement at turbulent flow become weak turbulence by the large magnetic field even where the flow state happens to be turbulent flow with the large buoyancy effect.

\section{Acknowledgment}

This work is performed with the partially support and under the auspices of the NIFS Collaborative Research Program (NIFS09KTBL007).

\section{References}

1) M. A. Abdou, APEX Team," Exploring novel high power density concepts for attractive fusion systems," Fusion Eng. Des., 45, 145 (1999).

2) Kasagi, N., Nishimura, M.,"Direct Numerical Simulation of Combined Forced and Natural Turbulent Convection in a Vertical Plane Channel," Int. J. Heat Fluid Flow, 18[1], 88 (1997).

3) O. Iida, N, Kasagi.,"Direct Numerical Simulation of Unstably Stratified Turbulent Channel Flow,"ASME J. Heat Transfer, 119, 53-61 (1997).

4) S. Satake, H. Chikamasa, T. Kunugi, K. Takase," Direct numerical simulation of unstable stratified turbulent flow under a magnetic field," 9th Int. Symp. Fusion Nuclear Technology, Oct. 11 -Oct 16, Dalian, China, (2009). 